Published in:

Journal of The Electrochemical Society, 152 (8), E239-E246 (2005)

\title{
Study of Molecular Structure Dependence of the Phase Transition Spike Response of Viologens at an HOPG Electrode using Bis-viologen and Carboxylated Viologen
}

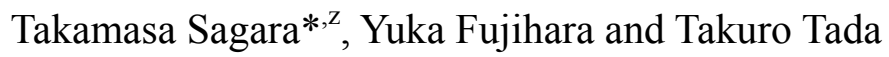

Department of Applied Chemistry, Faculty of Engineering, Nagasaki University, Bunkyo 1-14, Nagasaki 852-8521, Japan

Manuscript submitted November 1, 2004; revised manuscript received February 6, 2005. Available electronically June 27, 2005.

\section{For Figures, see pages 21-29.}

[footnote]

*Electrochemical Society Active Member

${ }^{z}$ E-mail: sagara@net.nagasaki-u.ac.jp 
Behavior of a bis-viologen and a carboxylated viologen in their faradaic phase transition at a highly-oriented pyrolytic graphite (HOPG) electrode between a gas-like adsorption layer and a two-dimensional condensed phase was studied. Bis-viologen (bis-V) possesses two 4,4'-bipyridinium moieties, and a carboxylated viologen ( $\mathrm{V}-\mathrm{COOH})$ does carboxyl groups at both terminals of the alkyl chains. The results of the voltammetric measurements were compared with heptyl viologen (HV) with a focus on the intermolecular lateral interaction among radical cations of the viologens in the condensed phase. It was found that the intermolecular attractive interaction between neighboring reduced forms of bis- $\mathrm{V}$ is stronger than that of $\mathrm{HV}$, indicating that intermolecular stacking of two radical cation moieties per molecule appears dominant over the negative effect of the possible chance of the presence of out-of-gear molecule pairs due to the mismatch in the neighboring molecule stacking upon the transition. The potential width of the bi-stable region of the phase transition for $\mathrm{V}-\mathrm{COOH}$ exceeded $120 \mathrm{mV}$ in an acidic medium due to the effective positive contribution of hydrogen bonding to the intermolecular attraction in the condensed phase on the hydrophobic basal plane of the HOPG electrode, while the contribution vanished in a basic medium. These results revealed that the voltammetric spike response of the phase transition can be a direct measure of the strength of two-dimensional intermolecular interaction on the electrode surface. Characteristics of the phase transition behavior of $\mathrm{V}-\mathrm{COOH}$ were also described in regards to temperature, concentration, and potential sweep rate dependencies. Implications of these characteristics were discussed in consideration of the dynamics of the assembling process of reduced forms of $\mathrm{V}-\mathrm{COOH}$ into the condensed layer. In the tests of the additives, only trimesic acid among the carboxylic acids used exhibited the activity as a hydrogen bonding inhibitor. 
An adsorption layer of viologens on a well-defined electrode surface frequently exhibits a pair of spike voltammetric peaks corresponding to one-electron redox reaction of the monolayer-level adsorption layer. ${ }^{1-19}$ This phenomenon has been understood as being a two-dimensional (2D) faradaic phase transition. One-electron reduction of viologen to mono-radical mono-cation is accompanied by the formation of the condensed phase. $^{3-8,12-14,17-19}$ The oxidized di-cation form of the viologen $\left(\mathrm{V}^{2+}\right)$ is usually highly soluble in water, while one-electron reduced counterpart, mono-radical mono-cation $\left(\mathrm{V}^{\bullet+}\right)$, is less or sparingly soluble in water. The reduced form tends to form a $\pi$-stacked dimer. Since the reduced form has higher adsorptivity on electrode surfaces, the redox reaction of the adsorption layer is observed at less negative potential than the bulk redox potential. This voltammetric wave is a sharp spike at $\mathrm{Hg}, \mathrm{HOPG}$, and some other electrodes ${ }^{1-19}$, showing the nature of the first order phase transition.

The 2D phase transition mechanism of an organic monolayer between ordered and disordered states is a topic of great current interest in surface science of the electrified interface. $^{20-29}$ A deeper understanding of the factors governing the transition is highly desirable not simply to obtain a molecular level view but also to apply it to sharp, multi-modal switching devices. In both faradaic and non-faradaic 2D phase transitions, the short-range attractive interaction with the nearest-neighbor molecules is usually the predominant factor. ${ }^{29-31}$ A certain magnitude of the interaction is a prerequisite of the occurrence of the first order phase transition. The magnitude of the interaction also determines the potential width of the bi-stable region of the transition.

We have described the features of faradaic phase transition of heptyl viologen (HV) at a basal plane HOPG electrode. ${ }^{12,14,19}$ The transition of HV takes place between an expanded (gas-like) adsorption layer of the oxidized form $\left(\mathrm{HV}^{2+}\right)$, which is in equilibrium with solution phase $\mathrm{HV}^{2+}$, and a $2 \mathrm{D}$ condensed monolayer of the reduced form $\left(\mathrm{HV}^{\bullet+}\right)$. At a HOPG | HV solution interface, HV exhibits a $13 \mathrm{mV}$ difference between anodic and cathodic transition potentials at room temperature. ${ }^{12,14,19}$ This spike peak separation corresponds to so-called bi-stable potential region, in other words, van der Waals loop, in connection with the S-shaped curve of the plot of the superficial fraction of the condensed phase as a function of the electrode potential. According to the classical view of the 
faradaic phase transition after Frumkin and Laviron, the peak separation is determined by the global interaction energy parameter, especially by the strong attractive intermolecular interaction in the condensed phase. ${ }^{32,33}$ However, this view is based on the mean-field approximation. This approximation is lately regarded as being not necessarily applicable to the representation of equilibrium phase transition. ${ }^{19,29,31,34-40}$ The analysis on a basis of a 2D lattice gas model has recently been accepted as being more adequate than the Frumkin model. In the lattice gas model, each molecule is ideally represented as a point or an isotropic sphere. Structural anisotropy and complexity is ignored and a single mode of intermolecular interaction is assumed. In sharp contrast, a viologen molecule bearing alkyl chains such as HV cannot be regarded as a point or sphere. The alkyl chains lie down flatly in the condensed film on the HOPG surface. Thus, the lateral intermolecular interaction in the condensed phase can be multi-modal and anisotropic. In addition to predominant stacking interaction among $\mathrm{V}^{\bullet+}$ moieties, chain-chain interaction and long-range electrostatic interaction should be taken into account. When the molecule possesses interactive terminal groups, molecular edge-to-edge interaction may also be of significance. For such a molecule, faradaic phase transition behavior has not been systematically discussed in relation to the multimodal interaction so far to our best knowledge.

The aim of this work is at the experimental clarification how the intermolecular interaction for a molecule possessing structural complexity and ability of multi-modal anisotropic nearest-neighbor interaction governs the voltammetric behavior, especially the width of the bi-stable potential region. The clarification of this point may help our modeling of the phase transition of organic molecules possessing an ability to interact with the nearest-neighbors through multiple interaction modes. We herein examine the phase transition behaviors of two viologens, bis-viologen (bis-V) and carboxylated viologen $(\mathrm{V}-\mathrm{COOH})$, in order for the effect of the lateral interactions to be highlighted. The former, possessing two viologen moieties in a molecule, was selected to see the effect the stacking interaction of radical cation moieties, and the latter, possessing carboxyl groups at the terminals of alkyl chains, was selected to see the effect of hydrogen bonding. We describe how the stacking and molecular edge-to-edge interaction works on the phase 
transition features using the results of the voltammetric and potential step measurements.

Presumable 2D molecular alignment structures of these viologens are schematically depicted in Fig. 1. In the case of bis- $\mathrm{V}$, the interaction between a pair of reduced molecules aligned in parallel (for example, molecules A and B in Fig. 1-a) includes $\pi-\pi$ stacking interaction between two pairs of radical cation moieties as well as chain-chain interaction. The stacking interaction energy per a bis-V molecule should be greater than mono-viologen HV (Fig. 1-c). But this happens only when positional shift of one of the molecules relative to the neighbors does not take place. The positional shift would produce a mismatch of the viologen moieties, like molecule $\mathrm{C}$ out-of-gear with molecule $\mathrm{B}$ in Fig. 1-a. In the case of $\mathrm{V}-\mathrm{COOH}$, hydrogen bonding between the carboxyl groups at the ends of alkyl chains would work significantly on a hydrophobic HOPG basal plane even in an aqueous phase (Fig. 1-b). The hydrogen bonding should not work in the $\mathrm{pH}$ region much greater than $\mathrm{p} K_{\mathrm{a}}$ of the carboxyl group. Hydrogen bonding is among the most important interaction in the molecular architecture. To mention just two recent examples: (1) hydrogen bonding is capable of forming a linear self-assembly of metal nanorods, ${ }^{42}$ and (2) coadsorption of bipyridyl compounds and stearic acid exhibits 2D molecular alignment patterns determined by hydrogen bonding structure. ${ }^{43}$ Hydrophobic nature of the HOPG surface may facilitate the formation of hydrogen bonding 2D network even in an aqueous medium, because water molecules as the hydrogen bonding competitor can be effectively expelled from the surface upon the adsorption of viologen. The effect of hydrogen bonding that is disclosed in this work may provide an indispensable guide in the design of a dynamic nano-assembly of molecules.

\section{Experimental}

1,1"-(1,4-butanediyl)bis[1'-(1-heptyl)-4,4'-bipyridinium] tetrabromide (Bis-V: Fig. 1-a) was synthesized by reacting 1-heptyl-4-(4-pyridyl)-pyridinium bromide (Aldrich, $3.0 \mathrm{~g}$, $8.9 \mathrm{mmol})$ with 1,4-dibromobutane $(0.26 \mathrm{~mL}, 2.2 \mathrm{mmol})$ in acetonitrile $(120 \mathrm{~mL})$ under reflux for $58 \mathrm{~h}$. Resulting precipitate was collected by filtration while hot and washed 
with hot acetonitrile. The dried product was further washed with acetonitrile at room temperature. The product was recrystallized from acetonirile-methanol to obtain a yellow crystal (yield: 18\%).

1,1'-bis(7-carboxyheptyl)-4,4'-bipyridinium dibromide (V-COOH Fig. 1-b) was prepared by reacting sublimated 4,4'-bipyridine $(1.0 \mathrm{~g}, 6.7 \mathrm{mmol})$ with 8-bromooctanoic acid $(4.7 \mathrm{~g}, 20 \mathrm{mmol})$ in DMF $(28 \mathrm{~mL})$ at $110^{\circ} \mathrm{C}$ for $13 \mathrm{~h}$. Resulting precipitate was washed with DMF and diethyl ether. The product was dispersed in $16 \% \mathrm{HBr}$ aqueous solution under sonication, and the liquid phase was vacuum evaporated. Addition of small amount of water and toluene followed by vacuum evaporation was repeated. The product was then recrystallized twice from acetone-water to obtain a light yellow crystal (yield: 64\%).

Water was purified through a Milli-Q Plus Ultrapure water system coupled with an Elix-5 kit (Millipore). Its resistivity was over $18 \mathrm{M} \Omega \mathrm{cm}$. All other chemicals were of reagent grade and used as received. A plate of HOPG (Panasonic graphite, PGX 04 or PGX 05: size $12 \mathrm{~mm} \times 12 \mathrm{~mm} \times 3 \mathrm{~mm}$-thickness) was connected perpendicularly with a copper pipe. To expose a fresh basal plane, the surface of the HOPG was peeled off by the use of $\operatorname{Scotch}^{\circledR}$ adhesive tape immediately before use. The HOPG electrode (basal plane electrode area: $A=1.44 \mathrm{~cm}^{2}$ ) was touched horizontally to the Ar gas | viologen solution interface and put in a hanging meniscus $(\mathrm{H}-\mathrm{M})$ configuration. The electrode potentials are all cited with reference to the $\mathrm{Ag}|\mathrm{AgCl}|$ saturated $\mathrm{KCl}$ reference electrode in this paper. All the electrochemical measurements were made using a Au wire coil counter electrode under an Ar gas (>99.998\%) atmosphere. Unless otherwise stated, the measurements were made at $22 \pm 2{ }^{\circ} \mathrm{C}$. Potential step measurements were made by the use of a potentiostat (HUSO, HECS 316/digital universal signal processing unit). The temperature dependence measurements were made by setting the reference electrode at the same temperature as the electrolyte solution.

\section{Results and Discussion}


A comparison of cyclic voltammograms for $\mathrm{HV}$, bis- $\mathrm{V}$, and $\mathrm{V}$-COOH.-Figure 2 shows cyclic voltammograms (CVs) for three viologens, $\mathrm{HV}$, bis- $\mathrm{V}$, and $\mathrm{V}-\mathrm{COOH}$. The midpoint potentials, $E_{\mathrm{a}, 1 / 2}=\left(E_{\mathrm{pa}}+E_{\mathrm{pc}}\right) / 2$, where $E_{\mathrm{pa}}$ is the anodic spike peak potential and $E_{\mathrm{pc}}$ is the cathodic one, are listed in Table 1 (inset in Fig. 2), together with the peak separation $\left(\Delta E_{\mathrm{p}}=E_{\mathrm{pa}}-E_{\mathrm{pc}}\right)$ at a potential sweep rate $(v)$ of $0.4 \mathrm{mV} \mathrm{s}^{-1}$. The reason why we used lower concentration for $\mathrm{V}-\mathrm{COOH}$ than for others will be described later. All three viologens showed a pair of spike peaks, corresponding to a one-electron transfer reaction per viologen unit, at less-negative potentials than the bulk redox reaction. The faradaic charge amount obtained from the peak area corresponded to the monolayer adsorption amount. Compared to $\mathrm{HV}$, the $\mathrm{CV}$ curve for bis-V was shifted to more negative with an increase of $\Delta E_{\mathrm{p}}$. The negative shift may be due to higher solubility of bis-dication form of bis- $\mathrm{V}$ than that of $\mathrm{HV}$, relatively stabilizing the soluble oxidized state. The greater value of $\Delta E_{\mathrm{p}}$ for bis-V than $\mathrm{HV}$ would be due to the effective enhancement of total attractive intermolecular interaction by the stacking of two radical cation moieties per molecule. Appearance of sharp spike indicates that the negative contribution of the mismatch (Fig. 1-a) to the sharp transition is minimal.

For V-COOH, the use of acidic medium ( $\mathrm{pH} 2.1$ ) may make its carboxylate groups be protonated. The much greater value of $\Delta E_{\mathrm{p}}$ compared to other two viologens indicates pronounced hydrogen bonding attraction among $\mathrm{V}-\mathrm{COOH}$ molecules in the condensed phase. The planar hydrogen bonding between two carboxyl groups, the energetically most stable configuration, can be formed on the hydrophobic HOPG electrode surface. If the $\mathrm{CV}$ response of $\mathrm{V}-\mathrm{COOH}$ is attributable to the first order phase transition, the value of $\Delta E_{\mathrm{p}}$ obtained in this work is one of the greatest one among a number of phase transitions for organic molecules so far observed.

Characteristics of room temperature phase transition behavior of $\mathrm{V}$-COOH at $0.1 \mathrm{mM}$ in an acidic medium. - The spike peak for $\mathrm{V}-\mathrm{COOH}$ is broader than other two viologens (Fig. 2). In order for the sharpness of the phase transition to be highlighted, we measured $\mathrm{CV}$ at various $v$ down to very slow rates as well as current transients in response to single and double potential steps. Note that all the results in this section were obtained at 
$22 \pm 2^{\circ} \mathrm{C}$ and at a $\mathrm{V}-\mathrm{COOH}$ concentration $(c)$ of $0.1 \mathrm{mM}$ in acidic media, $\mathrm{pH}=2.1$.

Figure 3 shows a plot of $E_{\mathrm{pa}}$ and $E_{\mathrm{pc}}$ of $\mathrm{CV}$ at various $v$. The peak separation was squeezed with the decrease in $v$ but was still over $120 \mathrm{mV}$ at $0.1 \mathrm{mV} \mathrm{s}^{-1}$. The magnitude of the change of peak potentials by the change of $v$ was comparable for anodic and cathodic processes, indicating that it is not the case that one of the processes is extremely slower than the other.

Figure 4-a shows typical current transients in response to single potential step perturbation. For both steps to negative and positive directions, a current hump followed the double-layer charging current. These features of the transient curves were typical for a nucleation-growth process. ${ }^{12}$ The slower decay of the current hump for the negative step is due to the fact that the final potential of the single potential step $\left(E_{\mathrm{f}}\right)$ is nearer to the transition potential for the negative step than that for the positive step.

Time-integrated charge $(Q)$ obtained from the single potential step chronoamperometry sequence was shown in Fig. 4-b. Note that $Q$ is the sum of faradaic and non-faradaic charges. An identical $Q-E_{\mathrm{f}}$ curve was obtained when the integration time period was set longer (10 s compared to $2 \mathrm{~s}$ for Fig. 4-b), indicating that $Q$ in Fig. 4-b covers the total charge to reach steady state at each $E_{\mathrm{f}}$. As for negative steps from fully-oxidized state at $-0.2 \mathrm{~V}$ to various $E_{\mathrm{f}}$, a steep increase of $Q$, which amounts to $11.5 \mu \mathrm{C} \mathrm{cm} \mathrm{cm}^{-2}$ corresponding to $1.2 \times 10^{-10} \mathrm{~mol} \mathrm{~cm}{ }^{-2}$ for a one-electron transfer process, was observed within a potential width of $8 \mathrm{mV}$. The charge amount of this almost stepwise increment is approximately the same as the charge obtained from the peak area of CV curves and also as the charge associated with the phase transition of HV. These results deny the occurrence of the condensation of the secondary layer onto the underlying first monolayer. As for positive steps from fully one-electron reduced state $(-0.70 \mathrm{~V})$ to various $E_{\mathrm{f}}$, the charge consumed in the full transition was nearly the same as that obtained by negative steps, whereas the potential width for transition was ca.14 $\mathrm{mV}$, much wider than that for the negative step. The potential width of transition $\mathrm{HV}$ was narrower than $1 \mathrm{mV},{ }^{12}$ indicating that phase transition of $\mathrm{V}-\mathrm{COOH}$ is less sharp than $\mathrm{HV}$. The broad $\mathrm{CV}$ peak for $\mathrm{V}-\mathrm{COOH}$ may be due to the presence of multiple but similar molecular organization structures. Figure 5 shows typical current transients as the response to double potential step perturbation. In 
Fig. 5-a, after nucleation of condensed phase was initiated at $E_{1}=-0.58 \mathrm{~V}<E_{\mathrm{c}}$ for a period of $5 \mathrm{~ms}$ by stepping the potential from $E_{0}=-0.20 \mathrm{~V}$, the growth of the condensed phase at $E_{2}=-0.49 \mathrm{~V}$ in the bi-stable potential region was clearly observed as the cathodic current following the anodic double layer charging current. Such a following growth of the condensed phase was observed in the range of $-0.50 \mathrm{~V}<E_{2}<-0.40 \mathrm{~V}$. The hole growth after its nucleation also was observed as shown in Fig. 5-b. Such a following hole growth was observed in the range of $-0.50 \mathrm{~V}<E_{2}<-0.40 \mathrm{~V}$.

The potential region, in which the following growth processes were observed, corresponds to the bi-stable potential region of the first order phase transition. This region actually falls in the potential region in between anodic and cathodic transition potentials in Fig. 4-b. The CV peak potentials at the measurable lowest $v$ (Fig. 3) were almost identical with the transition potentials obtained by potential step coulometry measurements (Fig. 4-b).

Taken together, although the voltammetric spike and the transition of $Q$ do not look as sharp as the phase transition of $\mathrm{HV}^{12}$, the behavior of $\mathrm{V}-\mathrm{COOH}$ phenomenologically meets the features of the first-order phase transition with nucleation-growth kinetics.

We will not go in more detail of the phase transition kinetics at this time. We must take into kinetic consideration solution phase diffusion, electron transfer rate, nucleation mechanism and rate, surface diffusion, growth rate, and domain shape and size. To see the extent of the contribution of the solution phase diffusion, we need to change the concentration of $\mathrm{V}-\mathrm{COOH}$ in a wide range as in our previous work ${ }^{14}$. As will be described in a later section, however, the transition behavior is changed at higher concentration than $0.20 \mathrm{mM}$ at room temperature. We will try to shed light in more depth of kinetics in future work. It is worthwhile to note that the increment of $Q$ at the transition potentials is identical in the range of the concentration from 0.10 to $0.18 \mathrm{mM}$, indicating the full transition to condensed phase takes place at $0.10 \mathrm{mM}$.

pH dependence of the transition behavior of $\mathrm{V}$-COOH.- - Figure 6-a shows typical CVs for $\mathrm{V}-\mathrm{COOH}(0.1 \mathrm{mM})$ at two far different $\mathrm{pH}$ at $v=50 \mathrm{mV} \mathrm{s}^{-1}$. In the alkaline solution $(\mathrm{pH}=11.1)$, the spike peak potentials were more negative compared to those for the 
representative $\mathrm{CV}$ in acidic solution $(\mathrm{pH}=2.1)$. The value of $\Delta E_{\mathrm{p}}$ was smaller in the alkaline solution. The anodic peak was less-sharp at $\mathrm{pH}$ 11.1. Both broadening and height decrement of the anodic peak started to occur around $\mathrm{pH} 9$ and were gradually more enhanced in more basic media. It was confirmed by the potential step experiments that the redox reaction holds the main features of the first order phase transition even in the basic solutions.

Figure 6-b represents the $\mathrm{pH}$ dependence of spike peak potentials at $v=50 \mathrm{mV} \mathrm{s}^{-1}$. Shifts of the spike peak potentials and $E_{\mathrm{a}, 1 / 2}$ to more negative direction, together with the steep decrease in $\Delta E_{\mathrm{p}}$, were observed in the $\mathrm{pH}$ range around 5.5-7.5. The shift of $E_{\mathrm{a}, 1 / 2}$ indicates the increase of solubility of $\mathrm{V}-\mathrm{COOH}$ due to its deprotonation in the basic solutions. The $\mathrm{pH}$ dependence of $\Delta E_{\mathrm{p}}$ indicates the occurrence of hydrogen bonding formation in acidic $\mathrm{pH}$ region. The lateral intermolecular edge-to-edge interaction due to the hydrogen bonding works in the acidic solutions in addition to the stacking of radical cation moieties and chain-chain interaction.

In the solution phase, the $\mathrm{p} K_{\mathrm{a}}$ value of an aliphatic carboxylic acid is around 4.8. Experimentally, the steepest shift of peak potentials occurred around pH 6.5 (Fig. 6-b), a higher $\mathrm{pH}$ than the solution $\mathrm{p} K_{\mathrm{a}}$ value. In the bulk solution phase, the hydrogen bonding formation between two carboxylic acid molecules seldom takes place even at $\mathrm{pH}<<\mathrm{p} K_{\mathrm{a}}$, because the bonding is always competitive to the hydrogen bonding with abundant water molecules. However, the hydrogen bonding formation occurs on the hydrophobic HOPG basal plane surface when carboxylate groups are protonated. The hydrogen bonding equilibrium should be taken into account only in a virtual surface layer of a thickness comparable to the $\mathrm{V}-\mathrm{COOH}$ molecular size. This is a typical case of the partition equilibrium between bulk solution and a thin surface layer, and hydrogen bonding formation can take place only in the latter phase. Then, at a given $\mathrm{pH}$, the fraction of protonated forms in the thin surface layer $\left(f_{\mathrm{s}}\right)$, which include both hydrogen bonded form and hydrogen-bonding free one, among the total amount of $\mathrm{V}-\mathrm{COOH}$ molecules should be much greater than the fraction in the bulk solution $\left(f_{\mathrm{b}}\right)$ as far as the partition equilibrium constant for carboxylic acid is nearly the same as that for carboxylate. This explains an increase of $\mathrm{p} K_{\mathrm{a}}$ value in the thin layer compared to the $\mathrm{p} K_{\mathrm{a}}$ in the bulk solution. In other 
words, the occurrence of hydrogen bonding formation as a precedent process shifts the proton dissociation equilibrium of carboxyl group to alkaline direction.

On a semi-quantitative basis, $f_{\mathrm{b}}$ can be expressed as a function of the proton concentration $\left(\left[\mathrm{H}^{+}\right]\right)$:

$$
f_{\mathrm{b}}=\frac{\left[\mathrm{H}^{+}\right]}{K_{a}+\left[\mathrm{H}^{+}\right]}
$$

On the other hand, $f_{\mathrm{s}}$ can be written as

$$
f_{\mathrm{s}}=\frac{\left[\mathrm{H}^{+}\right]}{\frac{K_{2}}{K_{1}} \frac{K_{\mathrm{a}}}{1+2 K_{\mathrm{H}} K_{1} c f_{\mathrm{b}}}+\left[\mathrm{H}^{+}\right]}
$$

where $K_{1}$ and $K_{2}$ are, respectively, the apparent partition constant of protonated form (carboxylic acid) and that of deprotonated form (carboxylate) from solution phase to the surface layer, $K_{\mathrm{H}}$ is the equilibrium constant for the formation of hydrogen bonded dimer in the surface layer, and $c$ is the total bulk concentration. Assuming that $K_{1}=K_{2}$ and $K_{\mathrm{a}}=$ $10^{-4} \mathrm{M}, f_{\mathrm{s}}$ is plotted in Fig. 7 using the term $K_{\mathrm{H}} K_{1} \mathrm{c}$ as a parameter. When $K_{\mathrm{H}}$ becomes greater, the $f_{\mathrm{s}}-\mathrm{pH}$ curves are shifted to more alkaline direction and become steeper. The tendency confirmed by this simple approximate calculation is in line with the experimental result (Fig. 6-b).

The $\mathrm{p} K_{\mathrm{a}}$ shift for adsorbed acid molecules on an electrode surface from the solution value was previously discussed by Dojlido and coworkers for acectylacetone ${ }^{44}$ and benzoic acid $^{45}$ at a $\mathrm{Hg}$ electrode. According to their thermodynamic approach taking the double layer effect into account, $\mathrm{p} K_{\mathrm{a}}$ value at the electrode surface is principally determined by the ratio of adsorption coefficients for acid and conjugate base forms. When the neutral acid adsorbs on the surface more strongly than the counterpart anionic conjugate base, the $\mathrm{p} K_{\mathrm{a}}$ value for the adsorbed species should be greater than that for the solution species. On one hand, the experimentally observed shift (Fig. 6-b) is in line with the above approach in 
regard to the stronger attraction of $-\mathrm{COOH}$ form to the $\mathrm{HOPG}$ surface than $-\mathrm{COO}^{-}$form. On the other hand, keeping in mind that the non-metallic HOPG shows much smaller interfacial capacity than a metallic electrode, such a great extent of shift of $\mathrm{p} K_{\mathrm{a}}$ for $\mathrm{V}-\mathrm{COOH}$ (approximately, $1.7 \mathrm{pH}$-unit) is difficult to be explained using their model. In addition, because the $\mathrm{CV}$ peak separation of the phase transition is an indicator of the intermolecular interaction, the double layer effect may explain only a minor part of the shift.

Another approach that includes explicitly the intermolecular interaction has been reported by Aoki, Kakiuchi, and corworkers ${ }^{46,47}$ for the case of a self-assembled monolayer (SAM) of a $\omega$-carboxylated alkanethiol. In the case of the SAM, predominant hydrogen bonding attraction as a short-range interaction over electrostatic repulsion as a long-range interaction has successfully explained the experimentally observed $\mathrm{p} K_{\mathrm{a}}$ shift to a larger value by more than $3 \mathrm{pH}$-units. This approach is in harmony with to our approach deducing Eq. [2], in which the stabilization of protonated form of $\mathrm{V}-\mathrm{COOH}$ on the electrode surface by hydrogen bonding is explicitly expressed by $K_{\mathrm{H}}$.

In order to analyze the $\mathrm{pH}$ dependence of the phase transition behavior of $\mathrm{V}-\mathrm{COOH}$ more quantitatively, estimation of the proton concentration near the adsorption layer may be helpful. However, the concentration should be a function of time in both voltammetric and potential step experiments. We need, therefore, a kinetic model of the phase transition to estimate the local proton concentration. The estimation of the proton concentration together with the kinetic modeling remains as a future work.

Concentration dependence of the transition behavior of $\mathrm{V}-\mathrm{COOH}$.-All the above mentioned measurements for $\mathrm{V}-\mathrm{COOH}$ were conducted at $c=0.1 \mathrm{mM}$. When $c$ was increased to $0.19 \mathrm{mM}$, a new anodic spike peak (peak $\mathrm{a}_{2}$ ) suddenly appeared at $160 \mathrm{mV}$ more negative potential than the peak potential of anodic peak $a_{1}$ (Fig. 8). At higher concentrations, this new anodic spike peak (peak $\mathrm{a}_{2}$ ) grew higher until original anodic spike peak $\mathrm{a}_{1}$ disappeared at $0.45 \mathrm{mM}$. The counterpart of both peaks $\mathrm{a}_{1}$ and $\mathrm{a}_{2}$ was found to be the cathodic peak $c_{1}$, since the appearance of peak $c_{1}$ was a prerequisite for the appearance of both peaks $a_{1}$ and $a_{2}$. In the concentration region where two anodic spike 
peaks coexist, the peak height ratio of $\mathrm{a}_{2} / \mathrm{a}_{1}$ was greater at higher $v$.

At higher concentrations, the concentration of radical cation in a close proximity to the HOPG surface increases within a shorter time period after the electrode potential becomes negative to the cathodic transition potential. Then, the radical cations are deposited on the surface randomly before the initiation of the formation of a highly aligned mono-molecular layer associated with hydrogen bonding interaction. This randomly deposited layer, the structure of which is far different from hydrogen bonded condensed layer, may be reoxidized upon reverse positive potential scan at nearly the same potential as the peak potential $\mathrm{c}_{1}$, because the stabilization due to the hydrogen bonding does not work.

It was also found that the onset of bulk reduction process, which takes place at more negative potential than the phase transition, was shifted to less-negative when peak $a_{2}$ is observed greater (not shown in the figure). The bulk redox reaction may be dependent of the state of the pre-formed adsorption layer as to whether the layer mainly produced at the cathodic peak $\mathrm{c}_{1}$ is the condensed phase $(c<0.19 \mathrm{mM})$ or randomly deposited phase $(c>$ $0.19 \mathrm{mM})$.

In order to follow the formation-reorganization process, the time course measurements of the film capacitance may be helpful if we use a metal electrode. In the case of HOPG, the experimental capacitance is always dominated by the space charge layer capacitance, which is in series with much greater film capacitance. In stead of the capacitance measurements, we are currently in preparation of the spectroelectrochemical tracking of the change of the state of the adsorption layer when peak $a_{2}$ is observed.

Temperature dependence of the transition behavior of $\mathrm{V}$-COOH.-The appearance of the new anodic spike peak (peak $a_{2}$ ) was found not only to be dependent of $c$ but also of temperature $(T)$ and $v$. For example at $T=32^{\circ} \mathrm{C}$ at $c=0.1 \mathrm{mM}$, the peak $\mathrm{a}_{2}$ was never observed in the range of $0.4 \mathrm{mV} \mathrm{s}^{-1}<v<1.0 \mathrm{~V} \mathrm{~s}^{-1}$ (Fig. 9-a). On the contrary, at $T=$ $10^{\circ} \mathrm{C}$ at the same $c$, the new anodic spike peak $\left(\mathrm{a}_{2}\right)$ was observed when $v>10 \mathrm{mV} \mathrm{s}^{-1}$ (Fig. 9-b). At lower $T$ and higher $v$, the well-organized condensed phase growth is incomplete, since random deposition is not prevented from its occurrence. 
Such dependence on $c, T$, and $v$ has never been observed for HV. In the case of $\mathrm{V}-\mathrm{COOH}$, the intermolecular interaction in the condensed phase is strengthened by hydrogen bonding in contrast to $\mathrm{HV}$. In addition to the transversely-lateral interaction between the $\mathrm{V}-\mathrm{COOH}$ molecules, viz. stacking interaction between radical cation moieties and chain-chain interaction, the molecular edge-to-edge longitudinal interaction, viz. hydrogen bonding formation, is effected simultaneously. The simultaneous presence of the two types of directional interactions allows the appearance of two possible adsorption layer 2D structures for the reduced forms, namely, one has intermolecular hydrogen bonding network and other does not. To form the former condensed layer, a time period is required for a molecule to look for its partner in the right alignment. But at low temperatures, lower solubility as well as slow surface diffusion of reduced molecule makes it not easy to find hydrogen bonding partners, especially at higher $v$. At low $T$ and higher $v$, random deposition of the reduced form takes place before hydrogen bonding network is formed because of the slow surface diffusion of the reduced form. Additionally, when too high transient concentration over saturation of reduced form is produced, randomly orientated deposition without the formation of hydrogen bonding network becomes dominant over the formation of 2D condensed layer with hydrogen bonding.

It is worth to note that the surface tension of $0.1 \mathrm{mM} \mathrm{V-COOH}$ solution at any $\mathrm{pH}$ was always almost the same as pure water. The film pressure at the air $\mid \mathrm{V}-\mathrm{COOH}$ solution interface, the surface tension of water from which that in the presence of $\mathrm{V}-\mathrm{COOH}$ was subtracted, was $0.5 \mathrm{mN} \mathrm{m}^{-1}$ at $c=1.0 \mathrm{mM}, 2.0 \mathrm{mN} \mathrm{m}^{-1}$ at $c=4.0 \mathrm{mM}$, and $4.5 \mathrm{mN} \mathrm{m}^{-1}$ at $c$ $=7.8 \mathrm{mM}$. These results indicate that the adsorptivity of $\mathrm{V}-\mathrm{COOH}$ to gas $\mid$ solution interface from solution phase is much less than HV. Therefore, it is likely that concentration of $\mathrm{V}-\mathrm{COOH}$ in the gas-like expanded phase (oxidized form of viologen) is not as high as HV, leading to deposition with an imperfect condensed film structure under certain conditions.

Effect of additives upon the transition behavior of $\mathrm{V}$-COOH. - Solution phase species which can compete with $\mathrm{V}-\mathrm{COOH}$ for hydrogen bonding formation may weaken the intermolecular interaction among $\mathrm{V}-\mathrm{COOH}$ molecules or even inhibit the formation of 
molecular alignment in the condensed phase. The test of the additives which may act as hydrogen bonding inhibitors is useful to support the hydrogen bonding formation and shed light on the nature of the bonding. We selected herein some carboxylic acids as the additives. The addition of acetic acid $(0.1 \mathrm{M})$, butylic acid (ca. $0.1 \mathrm{M})$, or valeric acid (near saturation) at $\mathrm{pH} 2.7$ in the presence of $0.3 \mathrm{M} \mathrm{KBr}$ did not affect the $\mathrm{CV}$ at all. These aliphatic acids are expected to act as a hydrogen bonding competitor. However, probably due to lower attraction to HOPG surface than the reduced form of $\mathrm{V}-\mathrm{COOH}$ or higher solubility, these acid molecules may be expelled from the surface proximity. On the other hand, addition of trimesic acid at saturated concentration at $\mathrm{pH} 2.0$ showed a pronounced effect. At room temperature, the new anodic spike peak $\mathrm{a}_{2}$ was observed together with a slightly negative shift of the anodic peak $\mathrm{a}_{1}$. When $T<20^{\circ} \mathrm{C}$, the original anodic peak $\mathrm{a}_{1}$ disappeared completely. Trimesic acid itself has a tendency to form a Gibbs layer at a gas | water (acid) interface. Trimesic acid molecules are not excluded from the HOPG surface region, acting effectively due to its three fold hydrogen bonding ability and resulting in the inhibition of the formation of the condensed phase of $\mathrm{V}-\mathrm{COOH}$ molecules with the aligned 2D structure.

Finally, addition of potassium acetate was tested to see whether or not carboxylate anion works as a counter ion of dication moiety of $\mathrm{V}-\mathrm{COOH}$ giving rise to the change voltammetric response. Addition of potassium acetate in the absence of $\mathrm{KBr}$ at $\mathrm{pH} 7.2$ and 11.4 did not affect $\mathrm{CV}$ at all even though multiple potential cycling was carried out to facilitate the counter anion $\left(\mathrm{Br}^{-}\right)$substitution by acetate ion. It is well known that the nature of counter anion greatly modifies the electrochemistry of viologens. ${ }^{48}$ The result of this test tells us that the extremely large peak separation for $\mathrm{V}-\mathrm{COOH}$ (Fig. 2) is at least not because of the function of carboxylate as the counter anion.

\section{Conclusion}

The faradaic phase transition processes of two viologens, bis-V and V-COOH (Fig. 1), were newly examined with a focus on the effect of the lateral intermolecular interaction 
among viologen radical cations in the condensed phase and compared with the results for HV. When the terminal carboxyl groups of $\mathrm{V}-\mathrm{COOH}$ are protonated, attractive hydrogen bonding interaction greatly widens the bi-stable potential region. In the case of bis-viologen, the widening of the bi-stable potential region is observed due to the increase of attractive stacking interaction between neighboring molecules each possessing two viologen radical cation moieties, in spite of the possibility of negative contribution of mismatch. It is revealed that, referring to $\mathrm{HV}$, the introduction of two carboxylic acid groups at both terminals of alkyl chains contributes more greatly to the widening of bi-stable potential region than the introduction of the second bipyridinium moiety. Presumably, hydrogen bonding can act on also the oxidized form as far as it is in contact with hydrophobic HOPG surface, while the staking works only for reduced form. The phase transition of $\mathrm{V}-\mathrm{COOH}$ is sensitive to its concentration, temperature, and the potential sweep rate. The importance of the fulfillment of suitable conditions to realize a well-ordered 2D condensed phase can be pointed out. Namely, disordered and random deposition of radical cation may tend to take place when initial concentration of radical cation is too high, temperature is low, or enough time to align the molecules is not given. Molecular design may enable us to achieve tailor-made phase transition behavior through the regulation of intermolecular interaction. In turn, this may provide us with abundant opportunities to examine intermolecular 2D interaction at an electrified HOPG surface.

In order to clarify the adsorption structure of the reduced form of $\mathrm{V}-\mathrm{COOH}$ and adsorption-reorganization process at a molecular level, direct observation of the structure using scanning probe microscopy or spectroscopic techniques is due course. Since the fulfillment of the strict condition for the appearance of the transition ${ }^{12}$ is difficult at present in the scanning probe microscopic experiments, we are currently underway of the preparation of spectroscopic measurements by IR or UV-visible reflectance. Condition dependent adsorption behavior of V-COOH should be shed light in more depth. Since the time scale of the reduction process is a key factor, kinetics of the transition remains to be explored. 


\section{Acknowledgements}

We thank the Ministry of Education, Culture, Sports, Science \& Technology of Japan for partial financial support to TS by a Grant-in-Aid for Scientific Research.

Nagasaki University assisted in meeting the publication costs of this article. 


\section{References}

1. A. Bewick, D. W. Cunningham, A. C. Lowe, Makromol. Chem. Macromol. Symp., 8, 355 (1987).

2. F. Kitamura, T. Ohsaka, K. Tokuda, J. Electroanal. Chem., 347, 371 (1993).

3. M. S. Maestre, R. Rodríguez-Amaro, E. Muñoz, J. J. Ruiz, L. Camacho, Langmuir, 10, 723 (1994).

4. R. Salas, M. Sánchez-Maestre, R. Rodríguez-Amaro, E. Muñoz, J. J. Ruiz, L. Camacho, Langmuir, 11, 1791 (1995).

5. R. Rodríguez-Amaro, J. J. Ruiz, L. Camacho, Rec. Res. Devel. Phys. Chem., 1, 309 (1997).

6. J. I. Millán, M. Sánchez-Maestre, L. Camacho, J. J. Ruiz, R. Rodríguez-Amaro, Langmuir, 13, 3860 (1997).

7. J. I. Millán, R. Rodríguez-Amaro, J. J. Ruiz, L. Camacho, Langmuir, 15, 618 (1999).

8. J. I Millán, R. Rodríguez-Amaro, J. J. Ruiz, L. Camacho, J. Phys. Chem. B, 103, 3669 (1999).

9. M. Tominaga, Abstracts of the 36th Joint Meeting of Kyushu Division of Chemical Societies, 4.63, 96 (1999).

10. K. Arihara, F. Kitamura, K. Nukanobu, T. Ohsaka, K. Tokuda, J. Electroanal. Chem., 473, 138 (1999).

11. K. Arihara, F. Kitamura, T. Ohsaka, K. Tokuda, J. Electroanal. Chem., 488, 117 (2000).

12. T. Sagara, S. Tanaka, Y. Fukuoka, N. Nakashima, Langmuir, 17, 1620 (2001).

13. J. I. Millán, J. J. Ruiz, L. Camacho, R. Rodríguez-Amaro, J. Electroanal. Chem., 497, 168 (2001).

14. T. Sagara, S. Tanaka, K. Miuchi, N. Nakashima, J. Electroanal. Chem., 524-525, 68 (2002).

15. K. Arihara, T. Ohsaka, F. Kitamura, Phys. Chem. Chem. Phys., 4, 1002 (2002).

16. K. Arihara, F. Kitamura, J. Electroanal. Chem., 550-551, 149 (2003).

17. J. I. Millán, J. J. Ruiz, L. Camacho, R. Rodríguez-Amaro, Langmuir, 19, 2338 (2003).

18. L. Gòmez, J. J. Ruiz, L. Camacho, R. Rodríguez-Amaro, J. Electroanal. Chem., 564, 
179 (2004).

19. T. Sagara, K. Miuchi, J. Electroanal. Chem., 567193 (2004).

20. R. deLevie, Chem. Rev., 88, 599 (1988).

21. C. Buess-Herman, in: J. Lipkowski, P. N. Ross (Eds.), Adsorption of Molecules at Metal Electrodes, VCH, New York, 1992, p. 77.

22. L. Stolberg, J. Lipkowski, in: J. Lipkowski, P. N. Ross (Eds.), Adsorption of Molecules at Metal Electrodes, VCH, New York, 1992, p. 171.

23. L. Pohlmann, C. Donner, S. Kirste, H. Baumgärtel, J. Phys. Chem. B, 101, 10198 (1997).

24. P. Nikitas, J. Electroanal. Chem., 446, 165 (1998).

25. N. J. Tao, in: J. Lipkowski, P. N. Ross (Eds.), Imaging of Surfaces and Interfaces, Wiley-VCH, New York, 1999, p 211.

26. D. Bizzotto, V. Zamlynny, I. Burgess, C. A. Jeffrey, H.-Q. Li, J. Rubinstein, R. A. Merril, J. Lipkowski, Z. Galus, A. Nelson, B. Pettinger, in: A. Wieckowski (Ed.), Interfacial Electrochemistry: Theory, Experiment, and Applications Marcel Dekker, New York, 1999, p 405.

27. C. Buess-Herman, S. Baré, M. Poelman, M. Van Kierken, in: A. Wieckowski (Ed.), Interfacial Electrochemistry: Theory, Experiment, and Applications Marcel Dekker, New York, 1999, p 427.

28. Th. Dretschkow, Th. Wandlowski, in: K. Wandelt, S. Thurgate (Eds.), Solid-Liquid Interfaces, Topics Appl. Phys. 85, Springer, Berlin, 2003, p. 259.

29. Th. Wandlowski, in: A. J. Bard, M. Stratmann (Eds.), Encyclopedia of Electrochemistry, Vol. 1, Wiley-VCH, Weinheim, 2003, p. 383.

30. U. Retter, H. Lohse, J. Electroanal. Chem., 134, 243 (1982).

31. U. Retter, J. Electroanal. Chem., 165, 221 (1984).

32. E. Laviron, J. Electroanal. Chem., 63, 245 (1975).

33. E. Laviron, in: A. J. Bard (Ed.), Electroanalytical Chemistry, vol. 12, Marcel Dekker, New York, 1982, p 53.

34. U. Retter, J. Electroanal. Chem., 236, 21 (1987).

35. R. Philipp, U. Retter, J. Dittrich, E. Müller, F. Kuschel, Electrochim. Acta, 32, 1671 
(1987).

36. U. Retter, V. Vetterl, J. Jursa, J. Electroanal. Chem., 274, 1 (1989).

37. Th. Wandlowski, J. Electroanal. Chem., 302, 233 (1991).

38. U. Retter, Electrochim. Acta, 41, 2171 (1996).

39. M. T. M. Koper, Electrochim. Acta, 44, 1207 (1998).

40. F. Berthier, B. Legrand, J. Creuze, R. Tétot, J. Electroanal. Chem., 561, 37 (2004).

41. S. Yin, C. Wang, X. Qiu, B. Xu, C. Bai, Surf. Interface Anal., 32, 248 (2001).

42. K. G. Thomas, S. Barazzouk, B. I. Ipe, S. T. S. Joseph, P. V. Kamat, J. Phys. Chem. B, 108, 13066 (2004).

43. B. Xu, Y. Chen, Q. Zeng, X. Qiu, C. Bai, Surf. Interface Anal., 32, 245 (2001).

44. J. Dojlido, Z. Galus, Lj. Jeftić, J. Electroanal. Chem., 62, 433 (1975).

45. J. Dojlido, M. Dmowska-stańczak, Z. Galus, J. Electroanal. Chem., 94, 107 (1978).

46. K. Aoki, T. Kakiuchi, J. Electroanal. Chem., 478, 101 (1999).

47. T. Kakiuchi, M. Iida, S. Nakabayashi, K. Niki, Langmuir, 16, 5397 (2000).

48. T. Sagara, H. Maeda, Y. Yuan, N. Nakashima, Langmuir, 15, 3823 (1999). 


\section{Figures}

(a) bis-V

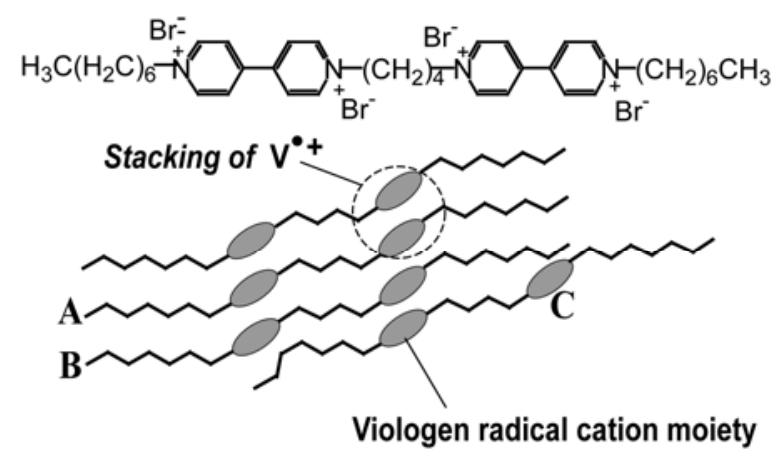

(b) $\mathrm{V}-\mathrm{COOH} \quad \mathrm{Br}$

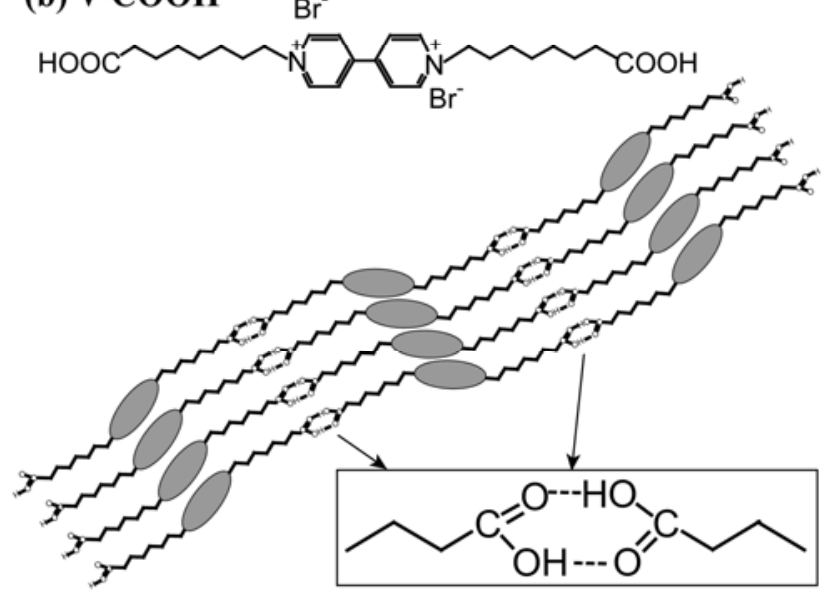

(c) $\mathrm{HV}$
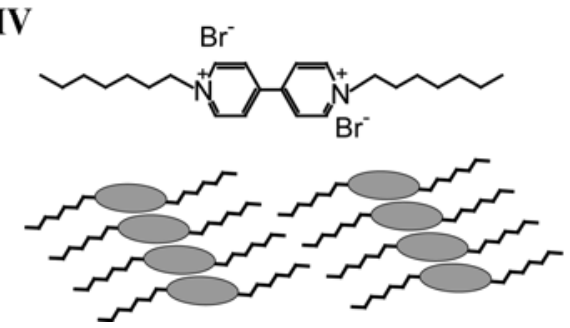

Figure 1. Schematic depiction of presumable molecular alignments of viologen cation radicals in the condensed phase on a HOPG electrode surface for bis-V (a), V-COOH (b), and HV (c). The ellipse represents a viologen radical cation moiety. The all-trans alkyl chain plane was assumed to be parallel to the electrode surface based on the reported energetic calculation by Bai and coworkers [41]. The molecular alignments of HV molecules were predicted in reference to the results of IR measurements reported by Arihara and coworkers [10]. 


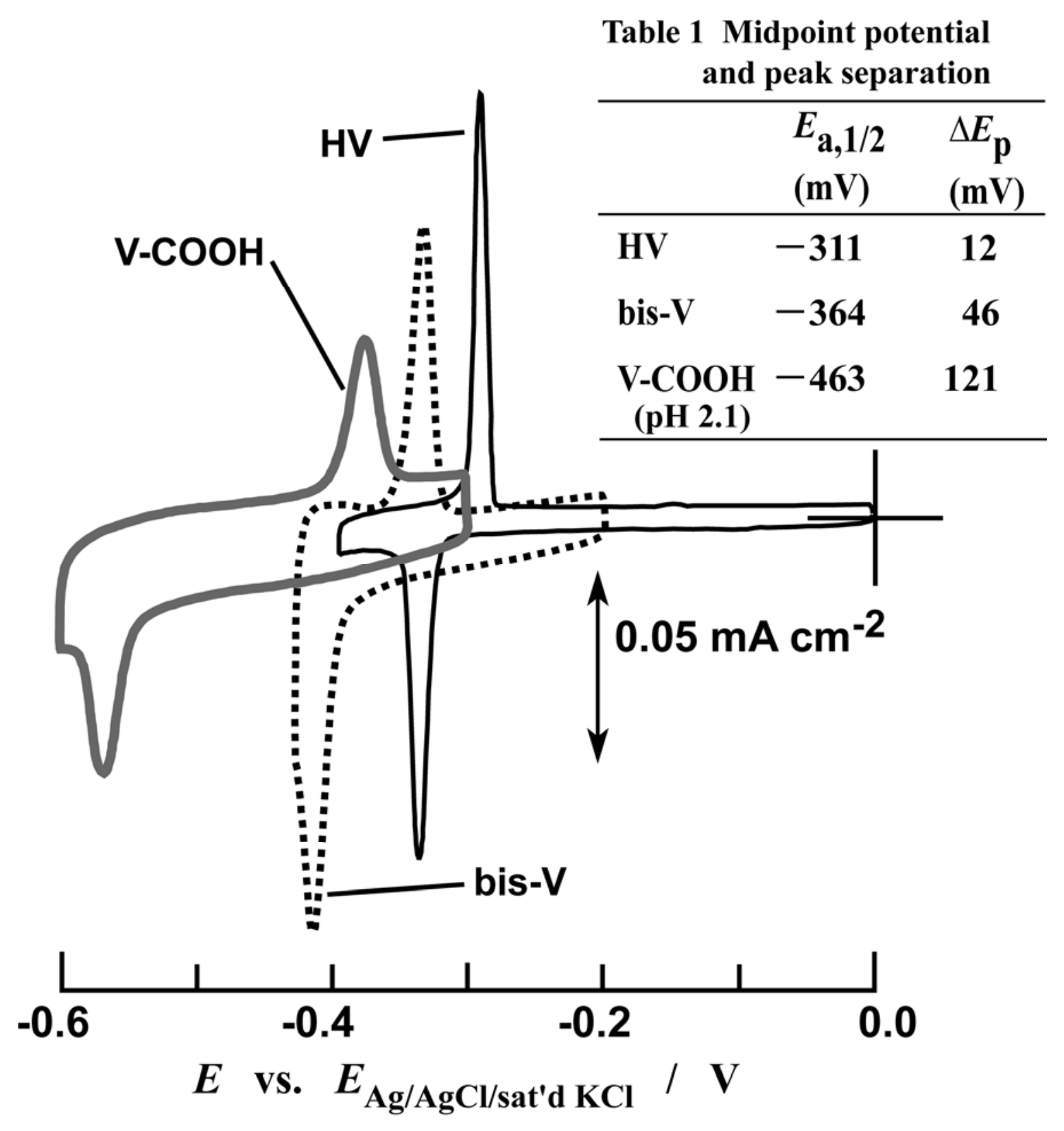

Figure 2. Cyclic voltammograms of $\mathrm{HV}$, bis-V, and $\mathrm{V}-\mathrm{COOH}$ at a HOPG electrode at $v=$ $80 \mathrm{mV} \mathrm{s}^{-1}$ at $23 \pm 1^{\circ} \mathrm{C}$. Solution compositions were as follows: $\mathrm{HV}$ and bis-V, $1.0 \mathrm{mM}$ in $0.3 \mathrm{M} \mathrm{KBr}$; V-COOH, $0.1 \mathrm{mM}$ in $0.3 \mathrm{M} \mathrm{KBr} \mathrm{pH}$-adjusted to be 2.1 by an addition of $\mathrm{HBr}$. Inset (Table 1): The values of $E_{\mathrm{a}, 1 / 2}$ and $\Delta E_{\mathrm{p}}$ obtained at $v=0.4 \mathrm{mV} \mathrm{s}^{-1}$. 


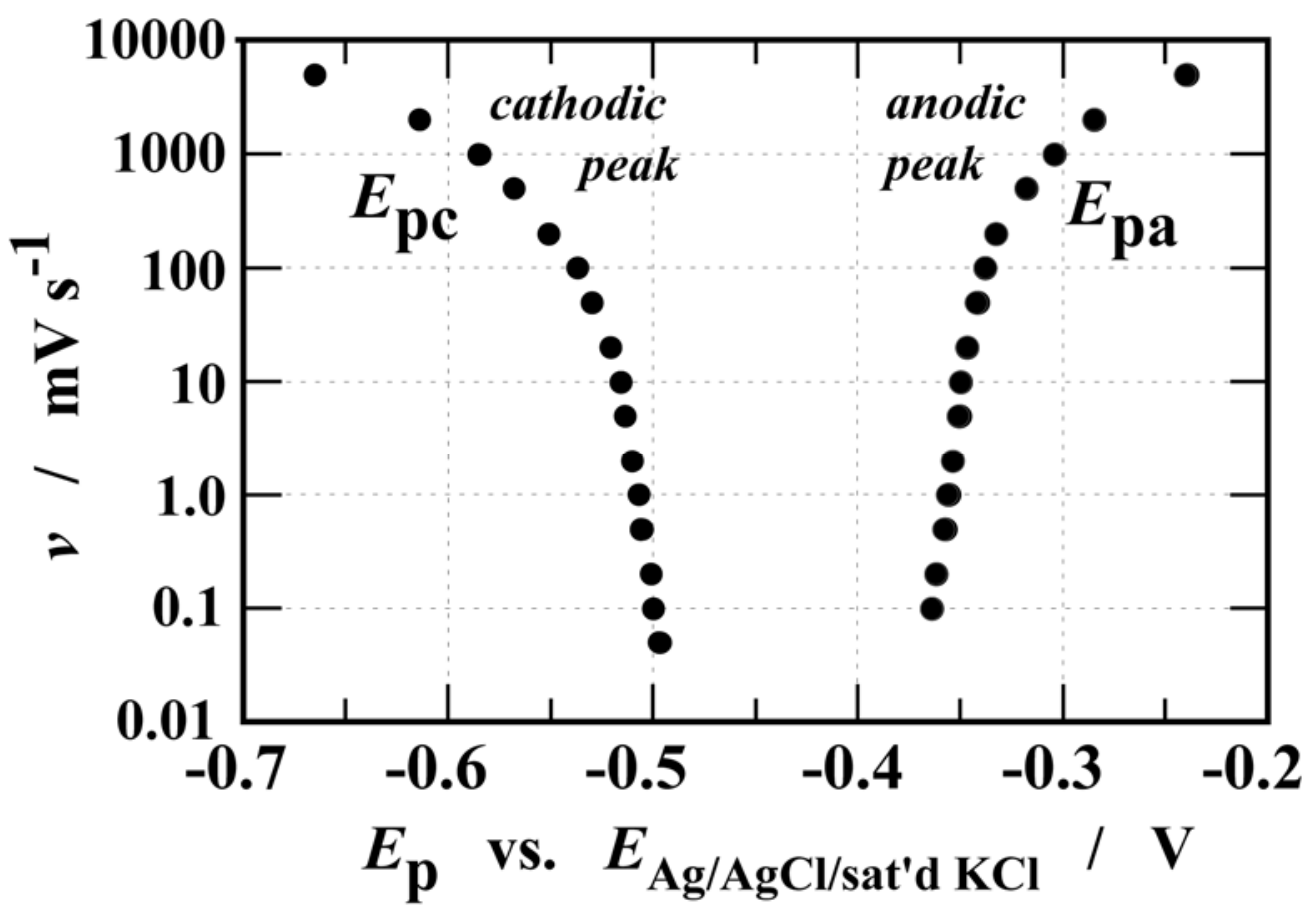

Figure 3. Plot of $E_{\mathrm{pa}}$ and $E_{\mathrm{pc}}$ for $\mathrm{V}-\mathrm{COOH}(0.1 \mathrm{mM}$ at $\mathrm{pH}=2.1$ in the presence of $0.3 \mathrm{M}$ $\mathrm{KBr}$ ) at a $\mathrm{HOPG}$ electrode at $22^{\circ} \mathrm{C}$. 
(a)

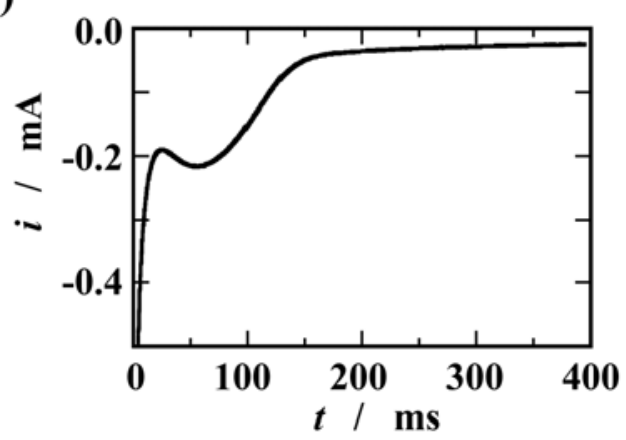

(b)

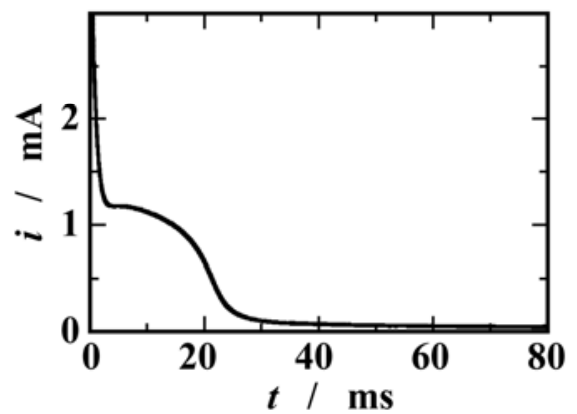

(c)

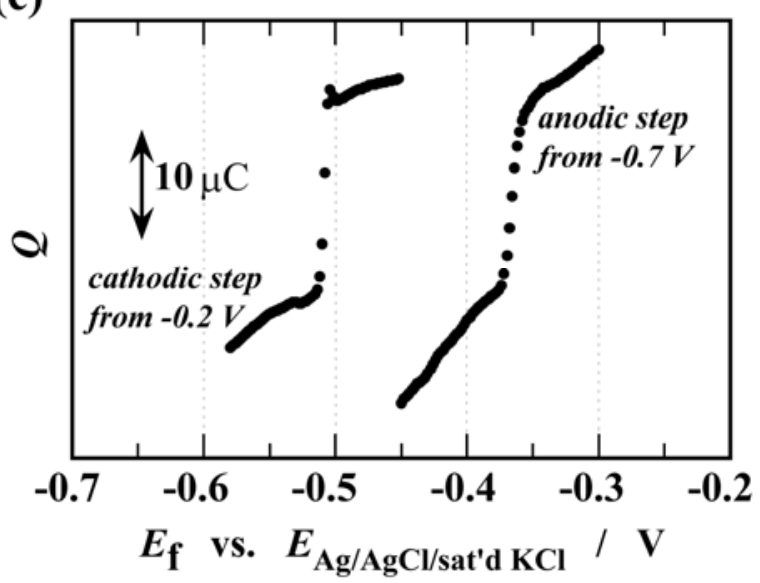

Figure 4. (a) and (b), Current transients in response to single potential step for V-COOH $(0.1 \mathrm{mM}$ at $\mathrm{pH}=2.1$ in the presence of $0.3 \mathrm{M} \mathrm{KBr})$ at a $\mathrm{HOPG}$ electrode at $22{ }^{\circ} \mathrm{C}$. (a) Negative step from the initial potential, $E_{\mathrm{i}}=-0.200 \mathrm{~V}$, to the final potential, $E_{\mathrm{f}}=-0.560 \mathrm{~V}$. (b) Positive step from $E_{\mathrm{i}}=-0.560 \mathrm{~V}$ to $E_{\mathrm{f}}=-0.200 \mathrm{~V}$. (c) Plot of the charge $(Q)$ obtained by the time-integration of the current transient curves against $E_{\mathrm{f}}$ obtained by potential step sequential measurements with various $E_{\mathrm{f}}$. The negative steps were given from $E_{\mathrm{i}}=-0.200 \mathrm{~V}$ to various $E_{\mathrm{f}}$ values, while positive steps were given from $E_{\mathrm{i}}=$ $-0.700 \mathrm{~V}$ to various $E_{\mathrm{f}}$ values. 


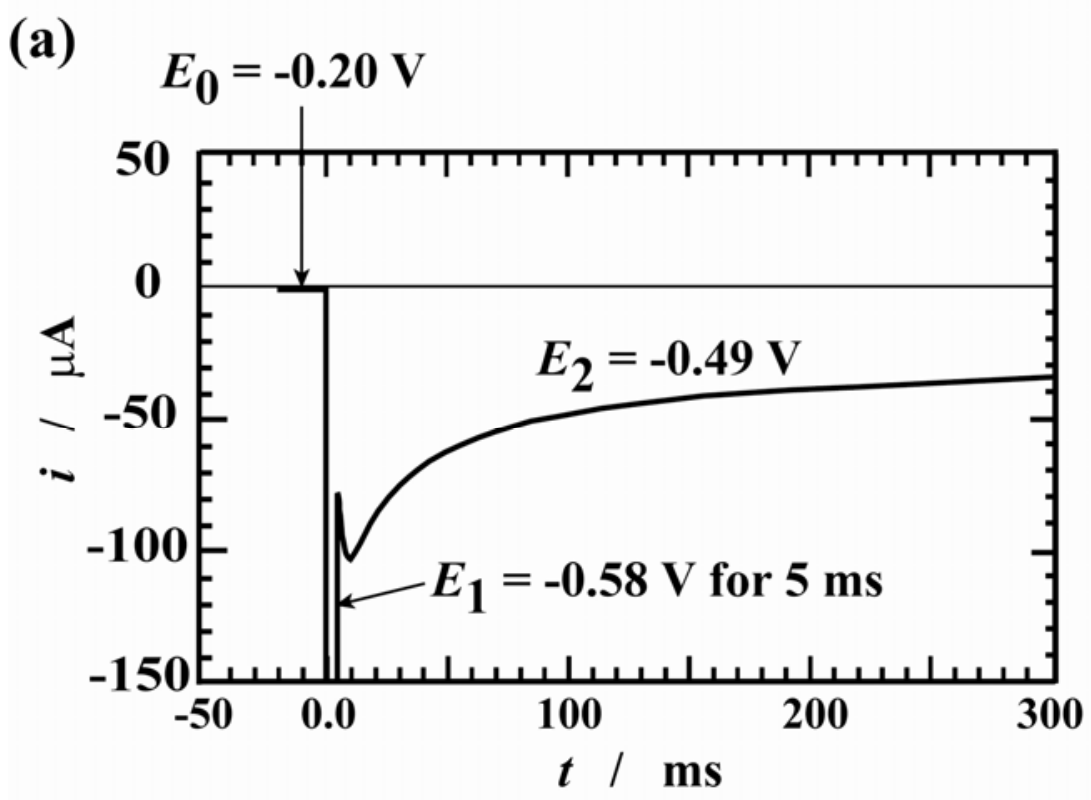

(b)

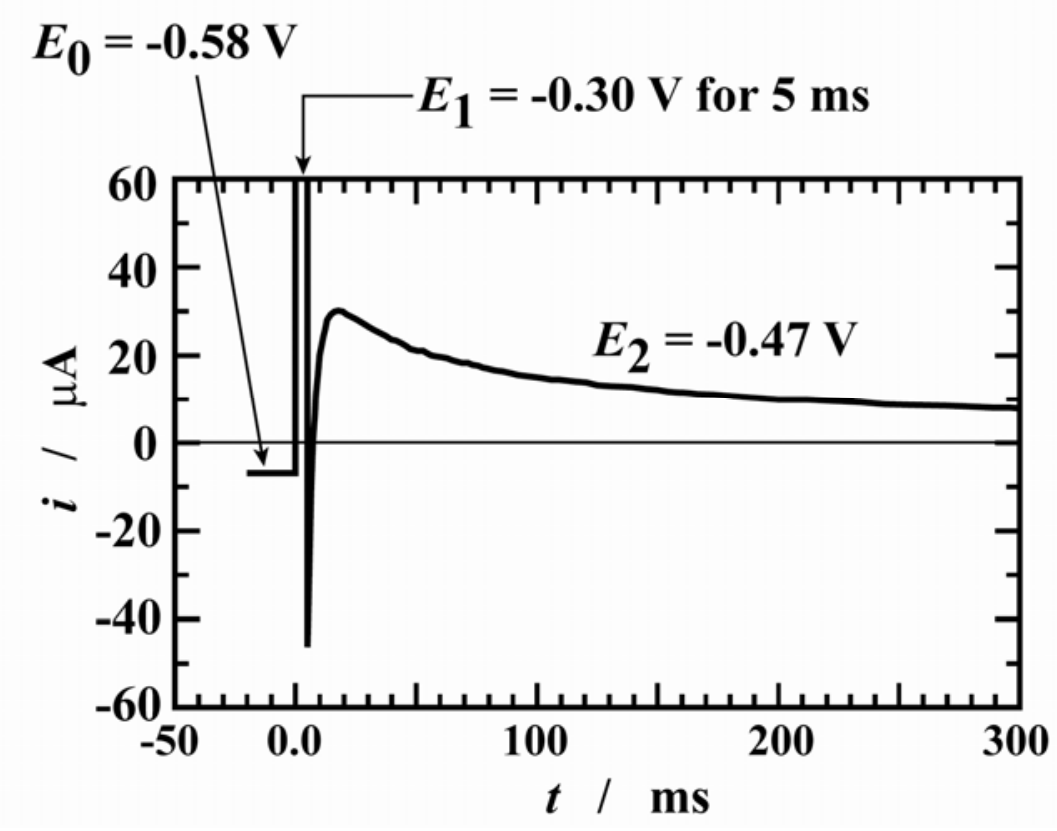

Figure 5. Typical current transients in response to double potential steps at a HOPG electrode with a solution composition of $0.1 \mathrm{mM} \mathrm{V-COOH}+0.3 \mathrm{M} \mathrm{KBr}(\mathrm{pH}=2.1)$. (a) The first step was given from the initial potential, $E_{\mathrm{i}}=-0.200 \mathrm{~V}$ to $E_{1}=-0.580 \mathrm{~V}$, and after spending at $E_{1}$ for a time period of $\tau_{1}=5.0 \mathrm{~ms}$, the second step to $E_{2}=-0.490 \mathrm{~V}$ was given. (b) $E_{\mathrm{i}}=-0.580 \mathrm{~V}, E_{1}=-0.300 \mathrm{~V}, \tau_{1}=5.0 \mathrm{~ms}$, and $E_{2}=-0.470 \mathrm{~V}$. 
(a)

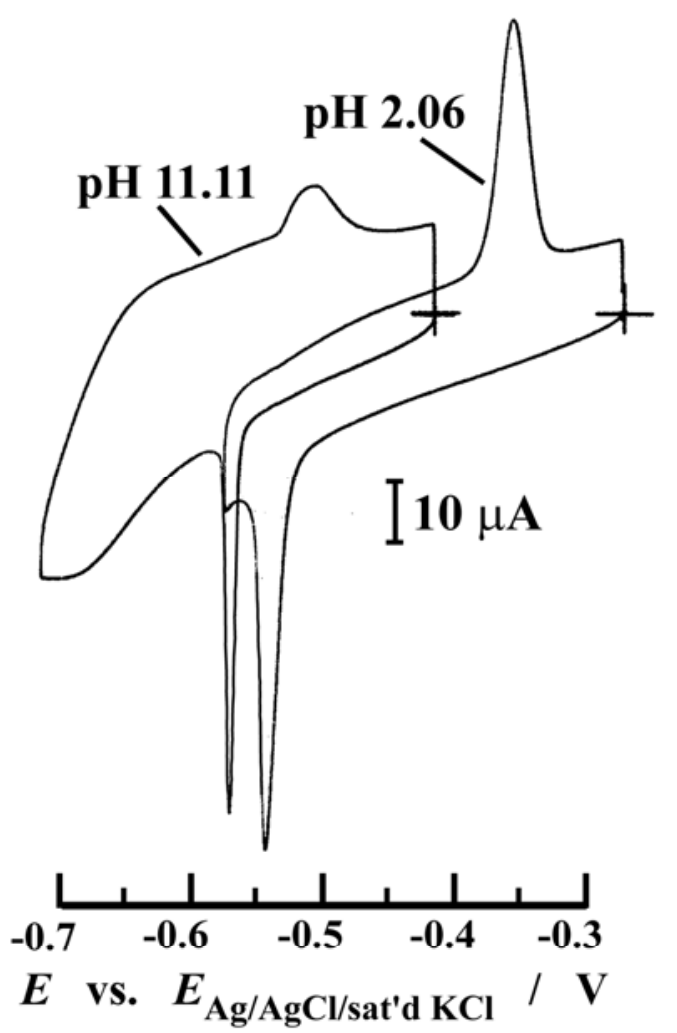

(b)

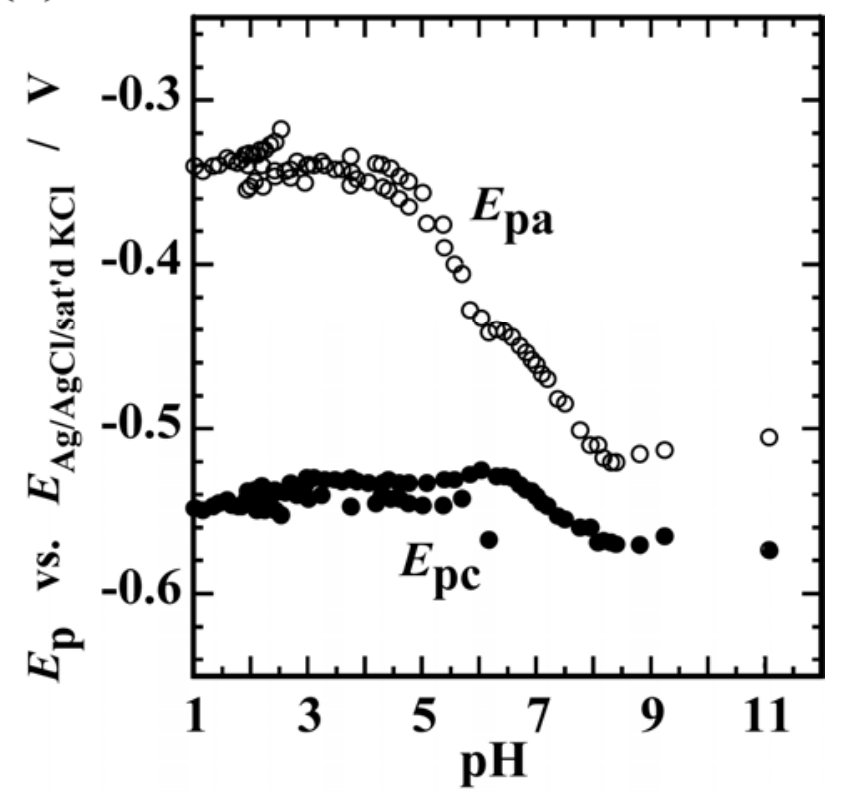

Figure 6. (a) Comparison of $\mathrm{CV}$ between at $\mathrm{pH}=2.1$ and 11.1 for a $\mathrm{HOPG}$ electrode | $0.1 \mathrm{mM} \mathrm{V-COOH}$ solution at $v=50 \mathrm{mV} \mathrm{s}^{-1}$. Adjustment of $\mathrm{pH}$ was made by adding $\mathrm{HBr}$, $\mathrm{KOH}$, or $\mathrm{K}_{2} \mathrm{HPO}_{4}$. No influence on $\mathrm{CV}$ with the presence of phosphate ions was observed. (b) The plot of $E_{\mathrm{pa}}$ and $E_{\mathrm{pc}}$ at a HOPG electrode in contact with $0.1 \mathrm{mM}$ $\mathrm{V}-\mathrm{COOH}$ solution at $v=50 \mathrm{mV} \mathrm{s}^{-1}$. 


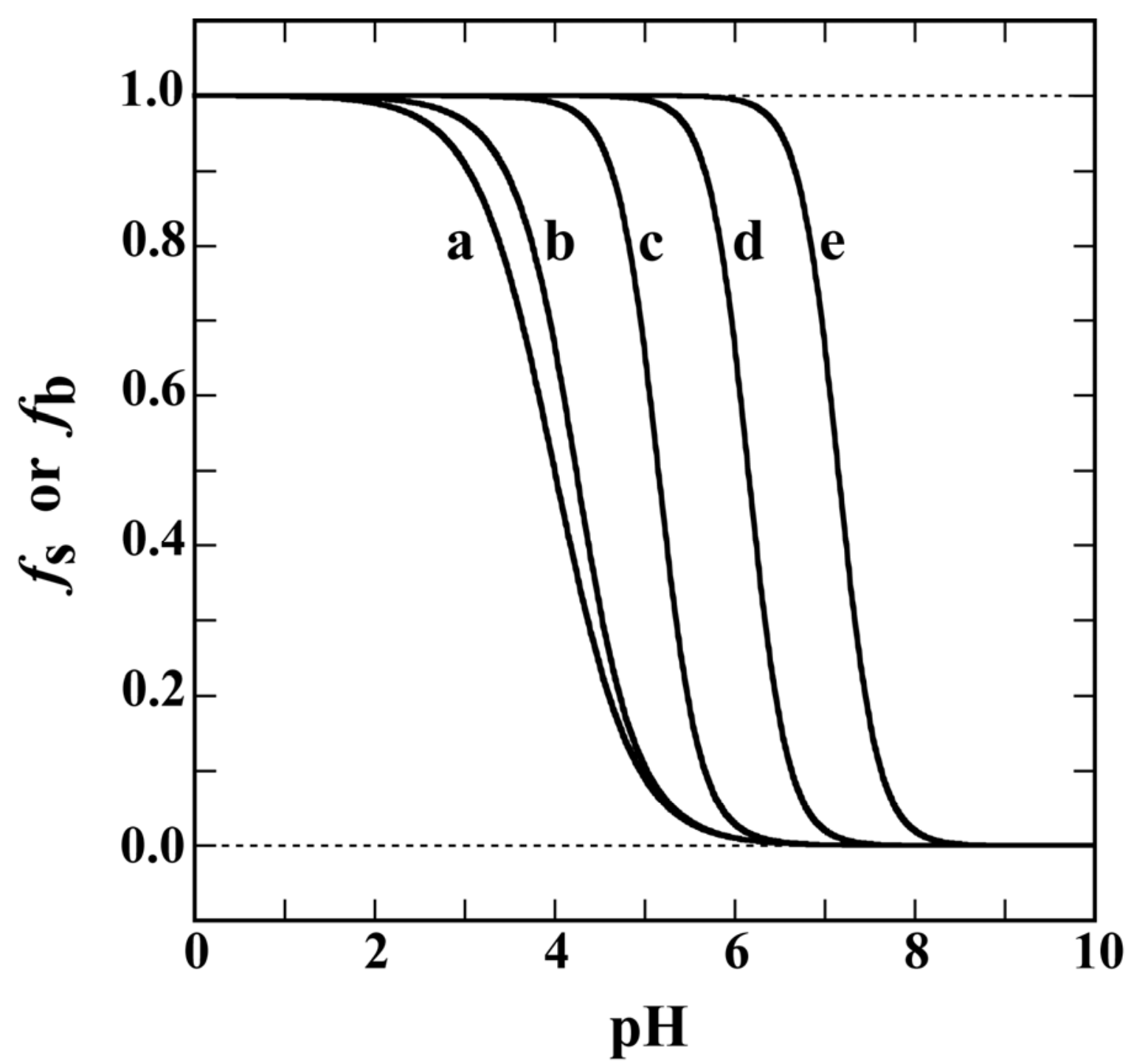

Figure 7. Plot of $f_{\mathrm{s}}$ calculated by the use of Eq. [2] as a function of solution $\mathrm{pH}$. Line a, $f_{\mathrm{s}}$ when $K_{\mathrm{H}} K_{1} C=0$ as well as $f_{\mathrm{b}}$; line b, $f_{\mathrm{s}}$ when $K_{\mathrm{H}} K_{1} c=1$; line c, $f_{\mathrm{s}}$ when $K_{\mathrm{H}} K_{1} c=10^{2}$; line $\mathrm{d}, f_{\mathrm{s}}$ when $K_{\mathrm{H}} K_{1} c=10^{4}$; line e, $f_{\mathrm{s}}$ when $K_{\mathrm{H}} K_{1} C=10^{6}$. 
(a) $0.4 \mathrm{mM} \mathrm{V-COOH}$
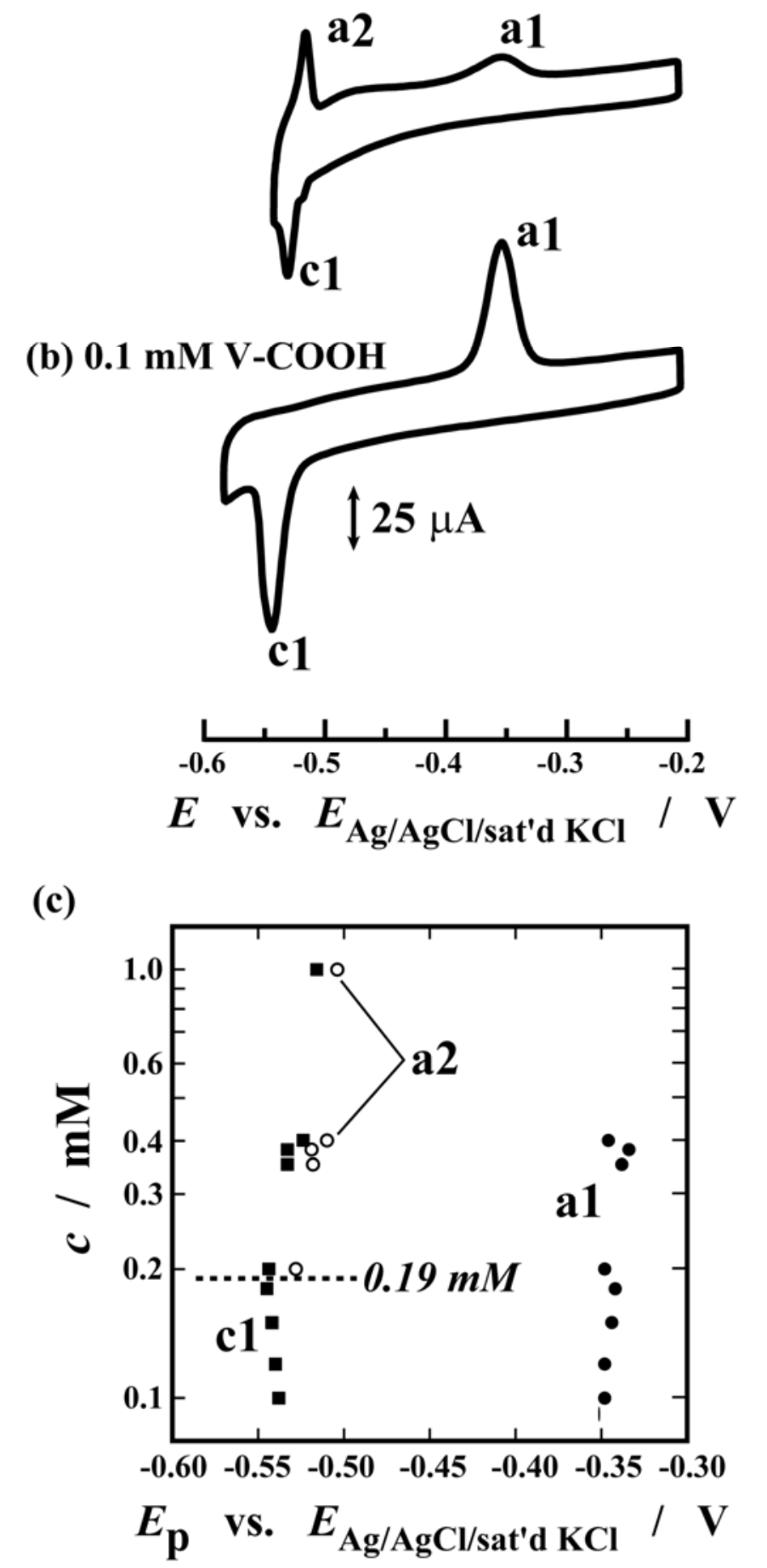

Figure 8. (a) and (b), CVs of a HOPG electrode in contact with $0.4 \mathrm{mM}$ (a) and $0.1 \mathrm{mM}$ (b) V-COOH solutions $(\mathrm{pH}=2.1)$ at $T=23^{\circ} \mathrm{C}\left(\mathrm{v}=80 \mathrm{mV} \mathrm{s}^{-1}\right)$. (c) $\mathrm{CV}$ peak potentials at $v=80 \mathrm{mV} \mathrm{s}^{-1}$ at various concentrations of $\mathrm{V}-\mathrm{COOH}(\mathrm{pH}=2.1)$. Closed circle, peak $\mathrm{a}_{1}$; open circle, peak $\mathrm{a}_{2}$; closed square, peak $\mathrm{c}_{1}$. 
(a) $32{ }^{\circ} \mathrm{C}$

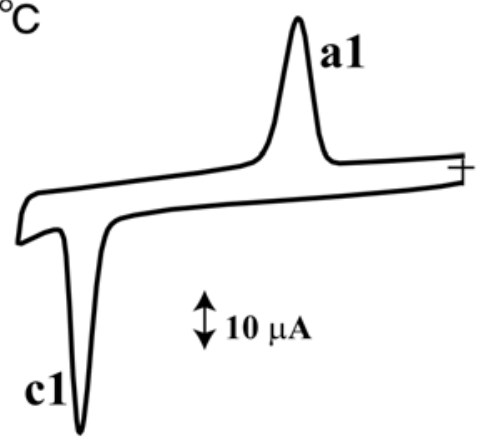

(b) $10^{\circ} \mathrm{C} \quad \mathrm{a2}$

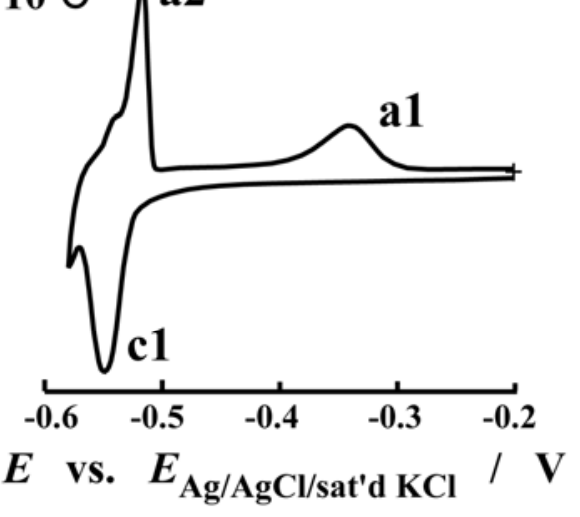

(c)

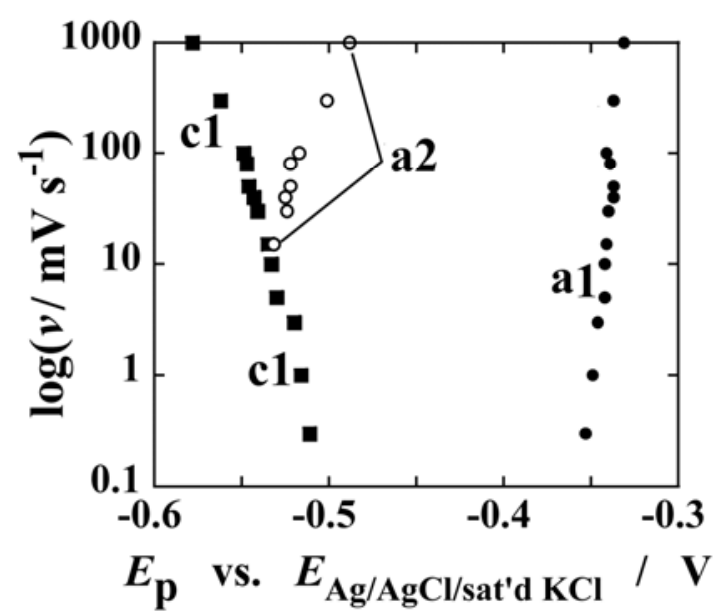

Figure 9. (a) and (b), CVs at two different temperatures at $100 \mathrm{mV} \mathrm{s}^{-1}$ for a HOPG electrode in contact with $0.1 \mathrm{mM} \mathrm{V}-\mathrm{COOH}$ solution at $\mathrm{pH} 2.1$. (a) at $32^{\circ} \mathrm{C}$, (b) at $10^{\circ} \mathrm{C}$. (c) Plot of $E_{\mathrm{p}}$ as a function of $v$ at $10^{\circ} \mathrm{C}$ for a $\mathrm{HOPG}$ electrode in contact with $0.1 \mathrm{mM}$ V-COOH solution at $\mathrm{pH}$ 2.1. Closed circle, peak $\mathrm{a}_{1}$; open circle, peak $\mathrm{a}_{2}$; closed square, peak $\mathrm{c}_{1}$. 